\title{
Análise do Sistema de Informação da Vigilância de Eventos Adversos Pós-Vacinação no Brasil, 2014 a 2016
}

\author{
Flávia Caselli Pacheco, ${ }^{1}$ Carla Magda Allan Santos Domingues, ${ }^{1}$ Ana Goretti \\ Kalume Maranhão, ${ }^{1}$ Sandra Maria Deotti Carvalho, ${ }^{1}$ Antonia Maria da Silva Teixeira, ${ }^{1}$ \\ Rui Moreira Braz, ${ }^{1}$ Renata Cristina Freitas Rebelo ${ }^{1}$ e Dirce Bellezi Guilhem²
}

Como citar Pacheco FC, Domingues CMAS, Maranhão AGK, Carvalho SMD, Teixeira AMS, Braz RM, et al. Análise do Sistema de Informação da Vigilância de Eventos Adversos Pós-Vacinação no Brasil, 2014 a 2016. Rev Panam Salud Publica. 2018;42:e12. doi: 10.26633/RPSP.2018.12

RESUMO Objetivo. Descrever as características das notificações de eventos adversos pós-vacinação (EAPV) no Sistema de Informação da Vigilância de EAPV (SI-EAPV) on-line nos primeiros 2 anos de operação do sistema.

Método. Foi realizado um estudo descritivo dos registros de EAPV notificados no SI-EAPV entre julho de 2014 e junho de 2016.

Resultados. Durante o período do estudo, foram registradas 24732 notificações. De 5570 municípios brasileiros, 2571 (46,2\%) realizaram notificação de algum EAPV. Entretanto, somente 1622 (6,6\%) notificações estavam encerradas no momento do estudo; dessas, 89,9\% não apresentaram gravidade. Entre as notificações encerradas, 19,7\% não tiveram o preenchimento da variável "atendimento médico" e 98,7\% não apresentaram registro de exames laboratoriais. As manifestações clinicas sistêmicas neurológicas foram as mais frequentes entre os eventos adversos graves encerrados, correspondendo a $59,5 \%$ dos sinais e sintomas. Em relação à idade, os maiores coeficientes de notificação foram registrados entre os menores de 4 anos. Conclusão. O SI-EAPV mostra-se útil no monitoramento da segurança das vacinas. Contudo, os municípios precisam ampliar a adesão ao sistema, bem como realizar as investigações e notificações dos EAPV, preenchendo a ficha de notificação de forma adequada e oportuna. O conhecimento sobre EAPV pode ser aplicado na prática dos serviços de vigilância em saúde, melhorando a segurança de utilização dos imunobiológicos.

Palavras-chave Vacinação; sistemas de informação; epidemiologia descritiva; saúde pública; Brasil.

Os eventos adversos pós-vacinação (EAPV) são ocorrências clínicas indesejadas ou não intencionais que ocorrem após a vacinação, sem que haja necessariamente uma relação causal com o uso da

\footnotetext{
Ministério da Saúde, Secretaria de Vigilância em Saúde, Coordenação Geral do Programa Nacional de Imunizações, Brasília (DF), Brasil. Correspondência: Flávia Caselli Pacheco, fpcaselli@gmail.com
}

vacina ou outro imunobiológico (1). Eventos desse tipo podem envolver um sintoma, uma doença ou um achado laboratorial anormal. Podem também ser relacionados à composição da vacina, à

\footnotetext{
2 Universidade de Brasília, Programa de PósGraduação em Ciências da Saúde, Brasília (DF), Brasil.
}

técnica usada em sua administração, aos próprios indivíduos vacinados - sendo a predisposição genética individual um importante fator no desenvolvimento de algumas desordens após a vacinação -, ou à coincidência com outros agravos (1-4).

Os EAPV não graves são mais frequentes e causam manifestações clínicas de leves a moderadas no local ou próximo ao sítio de administração da vacina (5-7). 
Por sua vez, os eventos adversos considerados graves, embora menos frequentes, requerem hospitalização por pelo menos 24 horas ou prolongamento de hospitalização já existente, produzem disfunção significativa ou incapacidade persistente (sequela), podem resultar em anomalia congênita e, quando apresentam risco de morte, exigem intervenção clínica imediata para evitar o óbito (1-3).

No Brasil, os registros de EAPV em indivíduos vacinados na rede pública são disponibilizados pelo Ministério da Saúde, por meio da Coordenação Geral do Programa Nacional de Imunizações (PNI), no Sistema de Informação da Vigilância de Eventos Adversos PósVacinação (SI-EAPV) $(8,9)$. O PNI iniciou a vigilância de EAPV em 1992, sendo a notificação compulsória instituída em 15 de julho de 2005 (3). Para os serviços da rede privada, a Agência Nacional de Vigilância Sanitária (ANVISA) disponibiliza o Sistema Nacional de Notificações para a Vigilância Sanitária (NOTIVISA) (10). Assim, os EAPV notificados no país procedem de duas fontes.

Para subsidiar o preenchimento das informações no sistema pelos profissionais, é disponibilizado um formulário próprio de investigação/notificação e o Manual de Vigilância dos EAPV, com informações sobre principais eventos relacionados às vacinas utilizadas na rede pública e instruções sobre a conduta a ser adotada ante a ocorrência desses agravos. Essa avaliação refere-se ao instrumento de notificação dos eventos no SI-EAPV, versão on-line (3).

Diante da importância do conhecimento sobre EAPV e sobre sua ocorrência para a tomada de decisões acertadas nos serviços e práticas de saúde a eles relacionados, este estudo teve como objetivo descrever as características das notificações de EAPV no SI-EAPV on-line nos primeiros 2 anos de operação do sistema.

\section{MATERIAIS E MÉTODOS}

Foi realizado um estudo descritivo com base nas notificações de EAPV registradas no Sistema de Informações do Programa Nacional de Imunizações (SI-PNI), módulo SI-EAPV, no período de $1^{\circ}$ de julho de 2014 (data de implantação da versão on-line do SI-EAPV) a 31 de junho de 2016. Foram analisadas todas as notificações registradas nesse período. Ressalte-se que, neste estudo, não foram incluídas as notificações realizadas no NOTIVISA, que é utilizado para os serviços de vacinação privados e se constitui em um sistema separado de notificação de EAPV.

A análise teve abrangência nacional, com dados por unidade federativa. Somente foram utilizados dados secundários, sem possibilidade de identificação de indivíduos, sendo portanto dispensada a aprovação por Comitê de Ética.

\section{Variáveis}

Foram analisados os casos de EAPV com e sem encerramento. As seguintes variáveis foram analisadas para os casos de EAPV encerrados: data do preenchimento da notificação; data de encerramento; tempo de encerramento (intervalo entre a notificação e o encerramento: 1 a 3 meses, 4 a 6 meses, 7 a 9 meses, 10 a 12 meses, 13 a 17 meses); faixa etária ( $<1$ ano, 1 ano, 2 anos, 3 anos, 4 anos, 5 a 9 anos, 10 a 14 anos, 15 a 19 anos, 20 a 29 anos, 30 a 39 anos, 40 a 49 anos, $\geq 50$ anos); sexo (masculino, feminino); raça/ cor (parda, branca, negra, amarela, indígena, não informado); tipo de EAPV (não grave, grave, erro de imunização, erro de imunização com evento adverso); atendimento médico (sim, não, ignorado); tipo de atendimento médico (ambulatorial, observação, hospitalização, não informado); registro de exames laboratoriais (sim, não); e evolução do caso (cura sem sequelas, cura com sequelas, em acompanhamento, não é EAPV, óbito, outros). $\mathrm{O}$ coeficiente de incidência foi calculado para cada 100000 habitantes. Os eventos adversos graves encerrados foram classificados de acordo com as manifestações clínicas (manifestações locais, manifestações clínicas sistêmicas em pele e mucosa, manifestações clínicas sistêmicas cardiovasculares, manifestações clínicas sistêmicas respiratórias, manifestações sistêmicas neurológicas, manifestações clínicas sistêmicas gastrintestinais, outras manifestações).

Para análise dos casos notificados de EAPV sem encerramento, foram utilizadas as seguintes variáveis: número de notificações por municípios; número de notificações por unidade federativa e coeficiente de incidência em 100000 habitantes.

As notificações de EAPV também foram analisadas com base no calendário nacional de vacinação do país e no número de doses aplicadas por faixa etária.
Para crianças com até 30 dias de vida, observou-se a vacina hepatite B. Nas crianças menores de 1 ano, foram consideradas as vacinas oral rotavírus humano, BCG, pentavalente, meningocócica C conjugada, pneumocócica 10 valente conjugada, poliomielite inativada e poliomielite oral. Para as crianças com idade entre 1 e 4 anos, foram consideradas as vacinas hepatite $\mathrm{A}$, tríplice viral e tetra viral. Para meninas entre 9 e 14 anos de idade considerou-se a vacina papilomavírus humano (HPV) quadrivalente; e entre os idosos ( $\geq 60$ anos), as vacinas consideradas foram influenza trivalente, hepatite $\mathrm{B}$ e febre amarela.

Os dados populacionais por faixa etária e unidade federativa, bem como o número de municípios por unidade federativa, foram gerados pelo Instituto Brasileiro de Geografia e Estatística (IBGE) e disponibilizados no site Datasus [Informações de Saúde (Tabnet) > Demográficas e socioeconômicas > População residente $>$ Censos]. Esses dados foram utilizados para cálculo do coeficiente de incidência de EAPV por faixa etária, por unidade federativa, e do número e proporção de municípios que realizaram notificações para EAPV. O número de doses aplicadas no período do estudo foi extraído do SI-PNI (Consultas > Informações $>$ Estatística $>$ Doses aplicadas).

\section{Controle de viés e análise estatística}

Para evitar o viés de informação, foram analisadas somente as notificações registradas a partir de $1^{\circ}$ de julho de 2014, período de implantação do sistema, mesmo que algumas unidades federativas tivessem realizado a retroalimentação dos casos suspeitos de EAPV no SI-EAPV. Essa medida foi adotada para evitar que municípios que fizeram a implantação do SI-EAPV posteriormente tivessem baixas notificações quando comparados aos que já estavam com o sistema implantado. A completude e a consistência dos registros foram avaliadas apenas para as notificações encerradas.

Os dados foram analisados por meio de estatística descritiva, utilizando-se o programa Microsoft Excel 2010.

\section{RESULTADOS}

Entre $1^{\circ}$ de julho de 2014 e 31 de junho de 2016, foram administradas 309072286 doses de imunobiológicos no Brasil. 
Desse total, 24732 casos foram notificados como suspeitos de terem desenvolvido EAPV no sistema público. Dos 5570 municípios brasileiros, 2571 (46,2\%) realizaram a notificação de algum EAPV, envolvendo as 27 unidades da federação. Os estados do Espírito Santo e de Minas Gerais, ambos na região Sudeste, apresentaram a maior proporção de municípios com notificação de casos suspeitos de EAPV (tabela 1). A tabela 2 mostra a distribuição das 24732 notificações de EAPV de acordo com imunobiológico e faixa etária.

A tabela 3 mostra a distribuição por unidade federativa dos 24732 casos suspeitos de EAPV registrados no SI-EAPV no período do estudo por unidade federativa e o número de casos encerrados por região/unidade federativa. Do total acumulado de EAPV notificados no Brasil, somente 1622 (6,6\%) estavam encerrados ao final do período do estudo.

Em relação às características das 1622 notificações com encerramento, observouse que o sexo feminino representou 949 $(58,5 \%)$ dos casos notificados e o masculino, 673 (41,5\%). Embora a raça/cor parda tenha sido a mais frequente $(36,6 \%)$ entre as notificações encerradas, vale notar que essa informação foi preenchida em apenas 67,5\% dos registros, como mostra a tabela 4 . Em relação às faixas etárias encontradas no estudo, os maiores coeficiente de incidência de notificações encerradas de EAPV em 100000 habitantes foram registrados entre os menores de 4 anos de idade. O coeficiente de incidência de EAPV em 100 mil habitantes foi o seguinte: 0,52 para $<1$ ano; 18,85 para idade de 1 ano; 15,83 para 2 anos; 3,24 para 3 anos; 1,07 para 4 anos; 0,89 para a faixa dos 5 aos 9 anos; 0,54 para a faixa de 10 a 14 anos; 0,28 para a faixa de 15 a 19 anos; 0,16 para a faixa de 20 a 29 anos; 0,21 para a faixa de 30 a 39 anos; 0,23 para a faixa de 40 a 49 anos; e 0,16 para idade $\geq 50$ anos.

Foram classificados como não graves $1295(79,8 \%)$ dos casos notificados. Houve atendimento médico em 854 $(52,7 \%)$ dos casos notificados; contudo, $319(19,7 \%)$ tiveram o preenchimento desta variável ignorada. No que tange ao tipo de atendimento, somente $83(5,1 \%)$ necessitaram de hospitalização, ao passo que $866(53,4 \%)$ não informaram o tipo de atendimento recebido (tabela 4 ).

Do total de 1622 notificações, 1601 $(98,7 \%)$ não apresentaram registro de

TABELA 1. Distribuição dos municípios com notificação de eventos adversos pósvacinação por unidade federativa, Brasil, julho de 2014 a junho de 2016

\begin{tabular}{|c|c|c|c|}
\hline \multirow{2}{*}{ Região e unidade federativa } & \multirow{2}{*}{ No. total de municípios } & \multicolumn{2}{|c|}{ Municípios com notificação } \\
\hline & & No. & $\%$ \\
\hline Norte & 450 & 106 & 23,6 \\
\hline Rondônia & 52 & 20 & 38,5 \\
\hline Acre & 22 & 13 & 59,1 \\
\hline Amazonas & 62 & 6 & 9,7 \\
\hline Roraima & 15 & 4 & 26,7 \\
\hline Pará & 144 & 22 & 15,3 \\
\hline Amapá & 16 & 3 & 18,8 \\
\hline Tocantins & 139 & 38 & 27,3 \\
\hline Nordeste & 1794 & 693 & 38,6 \\
\hline Maranhão & 217 & 58 & 26,7 \\
\hline Piauí & 224 & 44 & 19,6 \\
\hline Ceará & 184 & 94 & 51,1 \\
\hline Rio Grande do Norte & 167 & 18 & 10,8 \\
\hline Paraíba & 223 & 55 & 24,7 \\
\hline Pernambuco & 185 & 52 & 28,1 \\
\hline Alagoas & 102 & 43 & 42,2 \\
\hline Sergipe & 75 & 39 & 52,0 \\
\hline Bahia & 417 & 290 & 69,5 \\
\hline Sudeste & 1668 & 915 & 54,9 \\
\hline Minas Gerais & 853 & 575 & 67,4 \\
\hline Espírito Santo & 78 & 58 & 74,4 \\
\hline Rio de Janeiro & 92 & 44 & 47,8 \\
\hline São Paulo & 645 & 238 & 36,9 \\
\hline Sul & 1191 & 626 & 52,6 \\
\hline Paraná & 399 & 277 & 69,4 \\
\hline Santa Catarina & 295 & 37 & 12,5 \\
\hline Rio Grande do Sul & 497 & 312 & 62,8 \\
\hline Centro-Oeste & 467 & 231 & 49,5 \\
\hline Mato Grosso do Sul & 79 & 36 & 45,6 \\
\hline Mato Grosso & 141 & 57 & 40,4 \\
\hline Goiás & 246 & 137 & 55,7 \\
\hline Distrito Federal & 1 & 1 & 100,0 \\
\hline Brasil & 5570 & 2571 & 46,2 \\
\hline
\end{tabular}

nenhum resultado laboratorial. A evolução ocorreu com cura sem sequelas em 1310 (80,8\%) de todas as notificações encerradas no período do estudo.

As manifestações clínicas sistêmicas neurológicas foram as mais frequentes entre os eventos adversos graves encerrados no sistema, com 97 ocorrências, correspondendo a 59,5\% dos sinais e sintomas. Essas foram seguidas por outras manifestações $(17 / 10,4 \%)$, manifestações locais $(16 / 9,8 \%)$ e por manifestações clínicas sistêmicas em pele e mucosa $(16 / 9,8 \%)$, gastrintestinais $(11 / 6,7 \%)$, respiratórias $(3 / 1,8 \%)$ e cardiovasculares $(3 / 1,8 \%)$. A variável manifestações clínicas foi preenchida em todos os 1622 registros.

O óbito foi registrado em seis $(0,4 \%)$ casos notificados e encerrados (tabela 5), sem ter tido necessariamente uma relação causal com a vacinação. Além disso, 11 casos $(0,7 \%)$ não foram confirmados como EAPV após investigação. O tempo de encerramento foi entre 1 e 3 meses em $772(47,6 \%)$ das notificações encerradas (tabela 4).

\section{DISCUSSÃO}

O presente estudo se propôs a avaliar os dados registrados nos 2 primeiros anos de operação do SI-EAPV on-line. Os municípios da região Sudeste foram, proporcionalmente, os que mais notificaram os EAPV. Por sua vez, em relação ao coeficiente de incidência de notificações para a população residente no estado, o Paraná (região Sul) foi o que apresentou o maior valor. No que 
TABELA 2. Distribuição das 24732 notificações de eventos adversos pósvacinação de acordo com imunobiológico e faixa etária, Brasil, julho de 2014 a junho de 2016

\begin{tabular}{|c|c|c|}
\hline Idade/vacina & No. doses & $\begin{array}{l}\text { No. de eventos adversos } \\
\text { pós-vacinação }\end{array}$ \\
\hline \multicolumn{3}{|l|}{30 dias } \\
\hline Hepatite B & 7646884 & 201 \\
\hline \multicolumn{3}{|l|}{$<1$ ano } \\
\hline Vacina oral rotavírus humano & 16665918 & 16870 \\
\hline BCG & 8914786 & 2645 \\
\hline Pentavalente & 25213402 & 29280 \\
\hline Meningocócica $\mathrm{C}$ conjugada & 16903460 & 1579 \\
\hline Pneumocócica 10 valente conjugada & 22557934 & 16615 \\
\hline Poliomielite inativada & 10017792 & 18112 \\
\hline Poliomielite oral & 1507213 & 1857 \\
\hline \multicolumn{3}{|l|}{1 a 4 anos } \\
\hline Hepatite A & 6519620 & 1201 \\
\hline Tríplice viral & 21527751 & 2925 \\
\hline Tetra viral & 5031850 & 1153 \\
\hline \multicolumn{3}{|l|}{9 a 14 anos } \\
\hline HPV quadrivalente & 112292 & 2251 \\
\hline \multicolumn{3}{|l|}{60 anos ou mais } \\
\hline Influenza trivalente & 1935992 & 795 \\
\hline Hepatite B & 1064493 & 83 \\
\hline Febre amarela & 324806 & 81 \\
\hline
\end{tabular}

concerne à gravidade, a maioria dos EAPV notificados foi não grave. O óbito ocorreu em um número reduzido de casos.

Entende-se que a variação no número de notificações registradas pelos municípios em cada unidade federativa pode ser resultado da baixa adesão ao registro de notificações; mas pode, também, ser resultado da implantação recente, em alguns estados, do módulo on-line do SI-EAPV, ou da ausência de recursos humanos treinados ou com conhecimento adequado para registro das notificações nessas unidades federativas.

Outro fator que pode interferir no registro dos casos suspeitos de EAPV está relacionado a problemas estruturais, como a dificuldade de concluir os relatórios para fechamento dos casos, dificuldades na conexão do SI-PNI/ SI-EAPV com a base de dados nacional e curto tempo disponibilizado pelo sistema para o preenchimento das fichas de notificação (caso o notificador demore com o preenchimento da ficha no sistema, terá que iniciar novamente a sessão). A mitigação desses problemas é de responsabilidade do Departamento de Informática do Sistema Único de Saúde (SUS) e deve merecer atenção.
Tais fatores podem comprometer a adoção de medidas adequadas e oportunas diante de EAPV (11), além de favorecerem o descrédito da vacinação por parte dos imunizados, dos seus familiares e dos próprios profissionais de saúde, que podem atribuir às vacinas eventos que não se devem a elas, ou que refletem apenas uma associação temporal $(12,13)$.

Em relação à proporção de notificações de EAPV, as cinco regiões do país apresentaram risco de EAPV. Contudo, esse risco distribuiu-se de forma desigual entre elas. Estudos realizados no Brasil também constataram distribuição heterogênea dos EAPV, com maiores incidências em regiões com desenvolvimento considerado alto, como as regiões Sudeste e Sul (14). Ao serem comparados os resultados deste estudo com outros estudos realizados nas unidades federativas, observou-se que, em 2011, o estado do Ceará notificou 402 EAPV (15) e Minas Gerais, 1449 (16). O estado de Pernambuco notificou 1167 casos entre 2009 e 2013 (17), e Rondônia registrou 516 casos entre 1998 e 2008 (18).

Mais de $90 \%$ dos casos notificados no SI-EAPV não foram encerrados até a data de atualização do banco de dados.
Outros estudos também encontraram um elevado número de notificações sem encerramento, o que leva a inferir que existem problemas na qualidade da informação do SI-EAPV - como erros na digitação, incompletude dos campos e falhas no fluxo de informações $(16,19)$. O tempo de encerramento da maioria dos casos foi inferior a 6 meses, porém alguns demandaram um tempo superior a 7 meses.

No que se refere ao sexo, embora a diferença tenha sido pequena, houve maior ocorrência de EAPV entre o sexo feminino. Quanto ao preenchimento da variável raça/cor, o valor encontrado não foi satisfatório quando comparado à completude de 98\% identificada em outros estudos brasileiros, embora o sistema de informação tenha sido diferente $(20,21)$. O preenchimento dessa variável fornece elementos importantes para um diagnóstico da situação de saúde dos indivíduos com notificação de EAPV, permitindo o planejamento de ações, programas e políticas para esta população.

Os EAPV mais frequentes foram classificados como não graves, reforçando a segurança das vacinas utilizadas no país. Por outro lado, a subnotificação dos eventos impede a generalização desse achado. A maior ocorrência de eventos não graves está de acordo com os achados de outros estudos realizados em diversos países (22-24).

Constataram-se registros de erros de imunização. Porém, sabe-se que eles podem ter ocorrido em função de erro programático, como resultado do preparo, manejo ou administração inadequados da vacina; ou podem ter sido coincidentes, ocorrendo após a vacinação por uma associação apenas temporal, sem nexo causal (25).

Aproximadamente um quinto dos casos notificados teve o preenchimento da variável "atendimento médico" ignorada. A proporção de casos hospitalizados foi baixa, o que remete, provavelmente, à baixa gravidade. Além disso, verificou-se também que a maioria dos casos evoluiu sem sequelas. O percentual de ocorrências que evoluíram ao óbito e a não gravidade dos casos foi semelhante ao divulgado em relatório dos Estados Unidos que, entre 2006 e 2010, relatou morte em $0,6 \%$ dos casos, $7,7 \%$ de eventos 
TABELA 3. Total de notificações de eventos adversos pós-vacinação por unidade federativa e proporção de casos encerrados, Brasil, julho de 2014 a junho de 2016

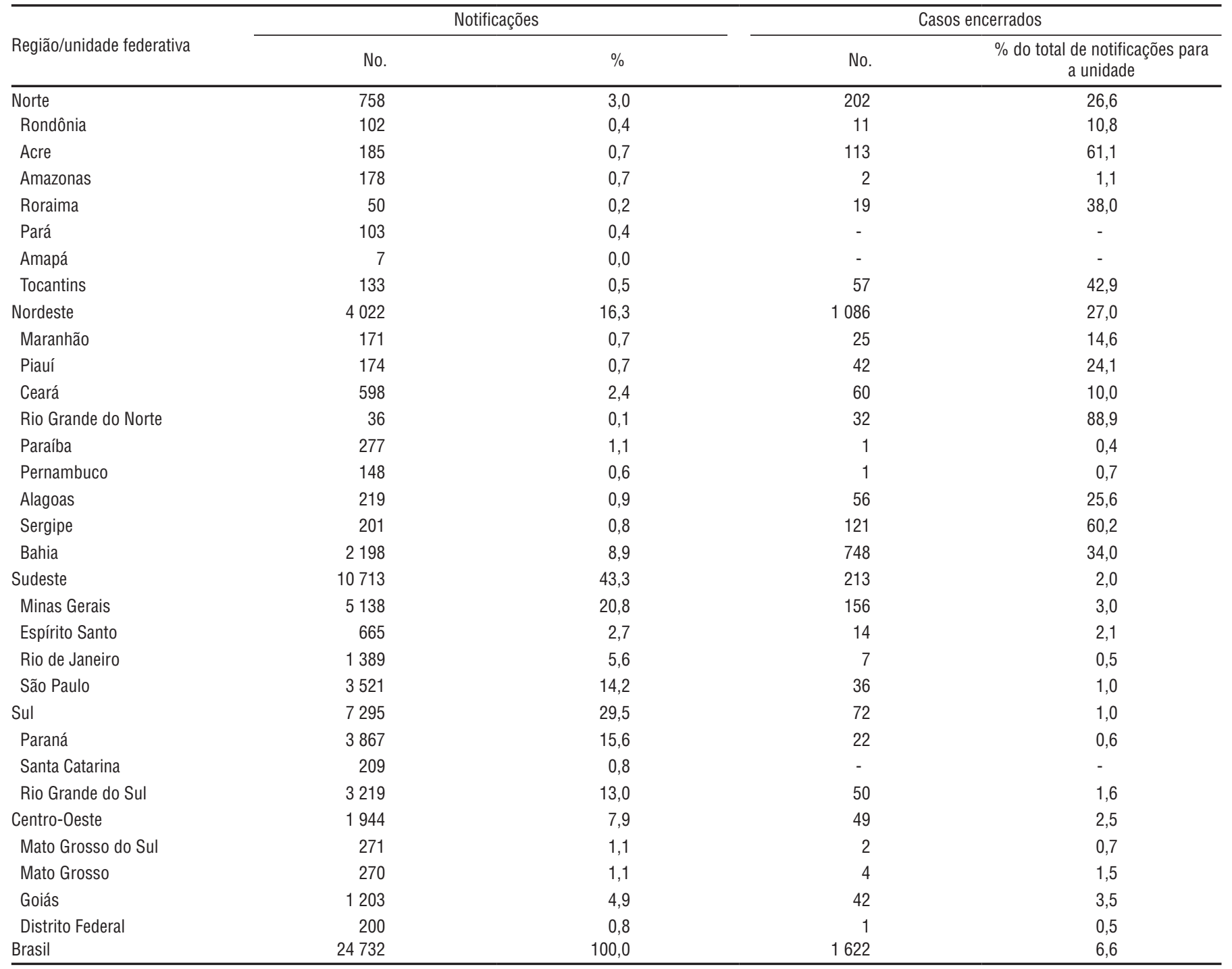

adversos graves não fatais e $91,7 \%$ de eventos não graves (26).

A baixa gravidade observada nos eventos não deveria inibir a realização de exames laboratoriais como hemograma, gota espessa, elementos anormais e sedimentoscopia, citologia do líquor, bacterioscopia e exames de diagnóstico por imagem em situações que requeiram este recurso (3). É indispensável uma criteriosa avaliação clínica e laboratorial dos casos para a busca rigorosa do diagnóstico etiológico e causal, com a finalidade de que o EAPV não seja atribuído à vacina sem fundamentação científica nem elucidação adequada do diagnóstico (27-29).
Ao se observar o maior coeficiente de incidência de EAPV por 100000 habitantes entre os menores de 4 anos de idade, é importante notar que, devido ao calendário nacional de vacinação adotado pelo PNI, o maior número de vacinas um total de 13 imunobiológicos - é disponibilizado pelas mais de 37 mil salas de vacinação do SUS para esta faixa etária. O mesmo foi relatado em crianças até 5 anos de idade, entre os anos de 1999 e 2008, em Cuba (30).

Informações equivocadas sobre EAPV podem diminuir a adesão à vacinação. Sabe-se que, diante da redução, ou até mesmo eliminação de doenças infecciosas imunopreveníveis, alguns indivíduos deixam de se preocupar com o risco de serem infectados, e cada vez mais se preocupam com os riscos associados às vacinas $(1,2)$. Porém, muitos países compreenderam a importância de reunir informações mais completas sobre os EAPV e os erros de imunizações e incorporaram em suas rotinas de trabalho sistemas de vigilância dos EAPV $(31,32)$.

Como limitações deste estudo destacam-se a ausência de notificações em todos os municípios do país, o número elevado de notificações sem encerramento e a falta de qualidade de informações registradas no SI-EAPV. Além disso, foram analisadas somente as notificações 
TABELA 4. Características das 1622 notificações de eventos adversos pós-vacinação com encerramento, Brasil, julho de 2014 a junho de 2016

\begin{tabular}{|c|c|c|}
\hline Característica & No. & $\%$ \\
\hline \multicolumn{3}{|l|}{$\overline{\text { Sexo }}$} \\
\hline Masculino & 673 & 41,5 \\
\hline Feminino & 949 & 58,5 \\
\hline \multicolumn{3}{|l|}{ Raça/cor } \\
\hline Parda & 593 & 36,6 \\
\hline Branca & 388 & 23,9 \\
\hline Negra & 91 & 5,6 \\
\hline Amarela & 18 & 1,1 \\
\hline Indígena & 5 & 0,3 \\
\hline Não informado & 527 & 32,5 \\
\hline \multicolumn{3}{|l|}{ Tipo de evento adverso pós-vacinação } \\
\hline Não grave & 1295 & 79,8 \\
\hline Grave & 163 & 10,0 \\
\hline Erro de imunização & 151 & 9,3 \\
\hline Erro de imunização com evento adverso & 13 & 0,8 \\
\hline \multicolumn{3}{|l|}{ Atendimento médico } \\
\hline $\operatorname{Sim}$ & 854 & 52,7 \\
\hline Não & 449 & 27,7 \\
\hline Ignorado & 319 & 19,7 \\
\hline \multicolumn{3}{|l|}{ Tipo de atendimento médico } \\
\hline Ambulatorial & 476 & 29,3 \\
\hline Observação & 197 & 12,1 \\
\hline Hospitalização & 83 & 5,1 \\
\hline Não informado & 866 & 53,4 \\
\hline \multicolumn{3}{|l|}{ Registro de exame laboratorial } \\
\hline Sim & 21 & 1,3 \\
\hline Não & 1601 & 98,7 \\
\hline \multicolumn{3}{|l|}{ Evolução do caso } \\
\hline Cura sem sequelas & 1310 & 80,8 \\
\hline Cura com sequelas & 26 & 1,6 \\
\hline Em acompanhamento & 78 & 4,8 \\
\hline Não é evento adverso pós-vacinação & 11 & 0,7 \\
\hline Óbito & 6 & 0,4 \\
\hline Outros & 41 & 2,5 \\
\hline Não informado & 150 & 9,2 \\
\hline \multicolumn{3}{|l|}{ Tempo de encerramento do caso (meses) } \\
\hline 1 a 3 & 772 & 47,6 \\
\hline 4 a 6 & 464 & 28,6 \\
\hline 7 a 9 & 307 & 18,9 \\
\hline 10 a 12 & 64 & 3,9 \\
\hline 13 a 17 & 15 & 0,9 \\
\hline
\end{tabular}

TABELA 5. Características dos seis óbitos em notificações de eventos adversos pós-vacinação com encerramento, Brasil, julho de 2014 a junho de 2016

\begin{tabular}{|c|c|c|}
\hline Idade & Vacina & Diagnóstico notificado no sistema ${ }^{a}$ \\
\hline 3 anos & Tríplice viral & Meningoencefalite \\
\hline 1 ano e 7 meses & Vacina oral de rotavírus humano & Obstrução do trato gastrintestinal \\
\hline 1 ano e 2 meses & Vacina oral de rotavírus humano & Dor abdominal agravada \\
\hline 13 anos e 3 meses & HPV quadrivalente & $\begin{array}{l}\text { Cefaleia, agitação mental, hipotonia, } \\
\text { irritabilidade e letargia }\end{array}$ \\
\hline 1 ano e 5 meses & Vacina oral de rotavírus humano & $\begin{array}{l}\text { Dor gastrintestinal, parada } \\
\text { cardiorrespiratória e choque séptico }\end{array}$ \\
\hline 15 anos e 5 meses & HPV quadrivalente & Encefalite \\
\hline
\end{tabular}

a 0s óbitos não têm necessariamente uma relação com a vacina. registradas a partir de $1^{\circ}$ de julho de 2014, período de implantação do sistema, mesmo que algumas unidades federativas tivessem realizado a retroalimentação dos casos suspeitos de EAPV no SI-EAPV ou tivessem mais registros de eventos notificados em outra fonte de informação. Finalmente, outra limitação é o fato de não terem sido incluídas informações do sistema de notificação da saúde suplementar.

Em conclusão, o conhecimento e a divulgação das análises do sistema de notificação de EAPV são importantes para a saúde pública, tanto no planejamento quanto na implementação de uma ação efetiva por parte de gestores, profissionais e população. Neste estudo, observou-se que os municípios precisam ampliar a adesão ao SI-EAPV, bem como preencher a ficha de notificação de forma mais adequada e oportuna. A solicitação dos exames laboratoriais deve ocorrer para subsidiar o encerramento adequado do caso suspeito, bem como a avaliação médica e a investigação minuciosa de cada caso.

Os problemas estruturais do sistema precisam ser sanados o mais brevemente possível pela área responsável no SUS.

Os serviços de imunização, nas três esferas de governo, devem divulgar os dados relacionados ao banco SI-EAPV por meio de boletins semanais, quinzenais ou mensais, adequando a linguagem de comunicação para os profissionais de saúde e para a população. É importante que todos, nas suas devidas competências, estejam aptos a detectar sinais e sintomas relacionados aos EAPV e busquem, o mais rapidamente possível, o serviço de saúde apropriado, para evitar complicações e risco de morte. O entendimento equivocado dos profissionais de saúde e da população em relação aos EAPV pode comprometer a adesão à vacinação e levar ao ressurgimento de doenças imunopreveníveis controladas ou eliminadas no país.

Conflitos de interesse. Nada declarado pelos autores.

Declaração. As opiniões expressas no manuscrito são de responsabilidade exclusiva dos autores e não refletem necessariamente a opinião ou política da RPSP/PAJPH ou da Organização PanAmericana da Saúde (OPAS). 


\section{REFERÊNCIAS}

1. World Health Organization (WHO). Global vaccine safety: adverse events following immunization (AEFI). Genebra: WHO; 2016. Disponível em: http://www. who.int/vaccine_safety/initiative/detection/AEFI/en/ Acessado em 10 de maio de 2016.

2. Center for Disease Control and Prevention (CDC). Vaccine Adverse Event Reporting System (VAERS). Atlanta: CDC; 2015. Disponível em: http://www.cdc.gov/vaccinesafety/ensuringsafety/monitoring/ vaers / Acessado em 10 de maio de 2016.

3. Brasil. Ministério da Saúde. Secretaria de Vigilância em Saúde. Departamento de Vigilância das Doenças Transmissíveis. Manual de vigilância epidemiológica de eventos adversos pós-vacinação. $3^{\mathrm{a}}$ ed. Brasília: Ministério da Saúde; 2014.

4. World Health Organization (WHO). Causality assessment of an adverse event following immunization (AEFI): user manual for the revised WHO classification. Genebra: WHO; 2013. Disponível em: http://www.who.int/vaccine_safety/publications/aevi_manual.pdf Acessado em dezembro de 2017.

5. Di Pasquale A, Bonanni P, Garçon N, Stanberry LR, El-Hodhod M, Tavares Da Silva F. Vaccine safety evaluation: practical aspects in assessing benefits and risks. Vaccine. 2016;34(52): 6672-80.

6. Macki M, Dabaja AA. Literature review of vaccine-related adverse events reported from HPV vaccination in randomized controlled trials. Basic Clin Androl. 2016;26:16.

7. Levi M, Donzellini M, Varone O, Sala A, Bechini A, Boccalini S, et al. Surveillance of adverse events following immunization with meningococcal group $\mathrm{C}$ conjugate vaccine: Tuscany, 2005-2012. J Prev Med Hyg. 2014;55(4):145-51.

8. Domingues CMAS, Teixeira AMS. Coberturas vacinais e doenças imunopreveníveis no Brasil no período 1982-2012: avanços e desafios do Programa Nacional de Imunizações. Epidemiol Serv Saude. 2013;22(1):9-27.

9. Brasil. Ministério da Saúde. Secretaria Executiva. Datasus. Sistema de Informações do Programa Nacional de Imunizações - Eventos Adversos PósVacinação. Manual do usuário. Brasília: Ministério da Saúde; 2003. Disponível em: http:/ / pni.datasus.gov.br/Download/ Eapv/EAPV-Manual.pdf Acessado em 8 de agosto de 2016.

10. Brasil, Ministério da Saúde. Portaria $n^{\circ}$ $1660 / 2009$. Diário Oficial da República Federativa do Brasil: Brasília; 2009. Disponível em http://www.anvisa.gov. br / hotsite / notivisa / Documentos / PORTARIA \%20No1660\%2022\%2007\%20 09.pdf?id $=23441 \&$ word $=$ Acessado em 5 de dezembro de 2017.

11. Global Advisory Committee on Vaccine Safety (GACVS), WHO Secretariat. Global safety of vaccines: strengthening systems for monitoring, management and the role of GACVS. Expert Rev Vaccines. 2009;8(6):705-16.

12. van Panhuis WG, Grefenstette J, Jung SY, Chok NS, Cross A, Lee BY, et al. Contagious diseases in the United States from 1888 to the present. N Engl J Med. 2013;369(22): 2152-8.

13. Waldman EA, Luhm KR, Monteiro SAMG, Freitas FRM. Surveillance of adverse effects following vaccination and safety of immunization programs. Rev Saude Publica. 2011;45(1):173-84.

14. Monteiro SAMG, Takano OA, Waldman EA. Avaliação do sistema brasileiro de vigilância de eventos adversos pós-vacinação. Rev Bras Epidemiol. 2011;14(3):361-71.

15. Moura ADA, Costa AS, Braga AVL, Bastos ECSA, Lima GG, Chaves ES. Vigilância de eventos adversos pós-vacinação no estado do Ceará, em 2011. Epidemiol Serv Saude. 2015;24(1):155-60.

16. Silva SS, Oliveira VC, Ribeiro HCTC, Alves TGS, Cavalcante RB, Guimarães EAA. Análise dos eventos adversos após aplicação de vacinas em Minas Gerais, 2011: um estudo transversal. Epidemiol Serv Saude. 2016;25(1):45-54.

17. Santos MCS, Pontes Netto VB, Andrade MS. Prevalência e fatores associados à ocorrência de eventos adversos pósvacinação em crianças. Acta Paul Enferm. 2016;29(6):626-32.

18. Cunha MP, Dórea JG, Marques RC, Leão RS. Vaccine adverse events reported during the first ten years (1998-2008) after introduction in the state of Rondonia, Brazil. Biomed Res Int. 2013;2013: 853083.

19. Araújo TME, Carvalho PMG, Vieira RDF. Análise dos eventos adversos pós-vacinais ocorridos em Teresina. Rev Bras Enferm. 2007;60(4):444-8.

20. Braz RM, Oliveira PTR, Reis AT, Machado NMS. Avaliação da completude da variável raça/cor nos sistemas nacionais de informação em saúde para aferição da equidade étnico-racial em indicadores usados pelo Índice de Desempenho do Sistema Único de Saúde. Saude Debate. 2013; 37(99):554-62.

21. Felix JD, Zandonade E, Amorim MHC, Castro DS. Avaliação da completude das variáveis epidemiológicas do Sistema de Informação sobre Mortalidade em mulheres com óbitos por câncer de mama na Região Sudeste - Brasil (1998 a 2007). Cienc Saude Coletiva. 2012. 17(4):945-53.

22. Jeetu G, Anusha G. Pharmacovigilance: a worldwide master key for drug safety monitoring. J Young Pharm. 2010;2(3): 315-20.

23. Moraes JC, Luna EJA, Grimaldi RA. Imunogenicidade da vacina brasileira contra hepatite B em adultos. Rev Saude Publica. 2010;44(2):353-9.
24. Freitas FRM, Sato HK, Aranda CMSS Arantes BAF, Pacheco MA, Waldman EA. Eventos adversos pós-vacina contra a difteria, coqueluche e tétano e fatores associados à sua gravidade. Rev Saude Publica. 2007;41(6):1032-41.

25. World Health Organization. Immunization safety surveillance: guidelines for immunization programme managers on surveillance of adverse events following immunization. $2^{a}$ ed. Manila: World Health Organization Regional Office for the Western Pacific; 2014. Disponível em: http:/ / www.wpro.who.int/topics / immunization_safety/Immunization SafetySurveillance.pdf Acessado em 3 de janeiro de 2017.

26. Miller ER, Haber P, Hibbs B, Broder K. Chapter 21: Surveillance for adverse events following immunization using the Vaccine Adverse Event Reporting System (VAERS). Em: VPD Surveillance Manual. $5^{a}$ ed. Surveillance for adverse events following immunization. Atlanta: Centers for Disease Control and Prevention; 2011. Pp. 21.1-11. Disponível em: https:/ /www. cdc.gov/vaccines/pubs/surv-manual/ chpt21-surv-adverse-events.pdf Acessado em 3 de janeiro de 2017.

27. Centers for Disease Control and Prevention (CDC). Understanding side effects and adverse events. Atlanta: CDC; 2015. Disponível em: http://www.cdc. gov/vaccinesafety/ensuringsafety/sideeffects/index.html Acessado em 3 de dezembro de 2017.

28. Tozzi AE. Field evaluation of vaccine safety. Vaccine. 2004; 22(15-16):2091-5

29. World Health Organization (WHO). AEFI Investigation. Genebra; WHO; 2016. Disponível em: http://www.who.int/vaccine_safety/publications/AEFI_ Investigation_Aide_Memoire.pdf Acessado em 3 de dezembro de 2017.

30. Galindo BM, Concepción D, Galindo MA, Pérez A, Saiz J. Vaccine-related adverse events in Cuban children, 1999-2008. MEDICC Rev. 2012;14(1):38-43.

31. Shimabukuro TT, Nguyen M, Martin D. Safety monitoring in the Vaccine Adverse Event Reporting System (VAERS). Vaccine. 2015;33(36):4398-405.

32. Zhou W, Pool V, Iskander JK, EnglishBullard R, Ball R, Wise RP, et al. Surveillance for safety after immunization: Vaccine Adverse Event Reporting System (VAERS) - United States, 19912001. MMWR Surveill Summ. 2003;52(1): $1-24$.

Manuscrito recebido em 20 de março de 2017. Aceito em versão revisada em 9 de setembro de 2017. 
ABSTRACT Objective. To describe the characteristics of vaccine adverse events (VAE) reports in the online VAE Reporting System (VAE-RS) after 2 years of operation.

Method. A descriptive analysis of VAE reports entered into the VAE-RS between July

Analysis of the Vaccine Adverse Event Reporting System in Brazil, 2014

to 2016

Keywords
2014 and June 2016 was performed.

Results. During the study period, 24732 VAE were reported. Of 5570 Brazilian municipalities, 2571 (46.2\%) reported at least one VAE; however, only 1622 (6.6\%) reports had been completed/closed at the end of the study period. Of these, $89.9 \%$ referred to mild VAE. Among the completed reports, $19.7 \%$ did not provide information on "type of medical care provided," and $98.7 \%$ had no information regarding laboratory tests. Systemic neurological symptoms were the most frequent serious VAE among closed reports $(59.5 \%$ of serious signs/symptoms). Concerning age, the highest VAE reporting coefficients were recorded for children aged $\leq 4$ years.

Conclusion. The VAE-RS is useful to monitor immunization safety. However, municipal services must increase adherence to the system and perform the required investigation and reporting of VAE, with timely and adequate completion of the VAE-RS form. Knowledge regarding VAE can be used in the daily routine of surveillance services, improving the safety of immunobiological agents.

Vaccination; information systems; epidemiology, descriptive; public health; Brazil.

RESUMEN

Análisis del Sistema de Información de Vigilancia de Eventos Adversos Posvacunación en Brasil, 2014 a 2016

Palabras-clave
Objetivo. Describir las características de las notificaciones de eventos adversos posvacunación (EAPV) en el Sistema de Información de Vigilancia de EAPV (SI-EAPV, un sistema en línea, durante los primeros 2 años de ejecución del sistema.

Método. Se realizó un estudio descriptivo de los registros de EAPV notificados en el SI-EAPV entre julio de 2014 y junio de 2016.

Resultados. Durante el período del estudio, se registraron 24732 notificaciones. De 5 570 municipios brasileños, 2571 (46,2\%) notificaron algún EAPV. Sin embargo, solamente 1622 (6,6\%) notificaciones estaban cerradas al momento del estudio; de ellas, el $89,9 \%$ no presentó gravedad. Respecto a las notificaciones cerradas, en el 19,7\% no fue anotada la variable "atención médica" y el 98,7\% no presentó registro de exámenes de laboratorio. Entre los eventos adversos graves cerrados, las manifestaciones clínicas sistémicas neurológicas fueron las más frecuentes, representado el 59,5\% de los signos y síntomas. En cuanto a la edad, los mayores coeficientes de notificación se registraron entre los menores de 4 años.

Conclusión. El SI-EAPV es útil para el monitoreo de la seguridad de las vacunas. Sin embargo, los municipios necesitan ampliar la adhesión al sistema, así como realizar las investigaciones y notificaciones de los EAPV, llenando la ficha de notificación de forma adecuada y oportuna. El conocimiento sobre EAPV puede ser aplicado en la práctica de los servicios de vigilancia en salud, mejorando la seguridad en la utilización de los productos inmunobiológicos.

Vacunación; sistemas de información; epidemiología descriptiva; salud pública; Brasil. 